\title{
Direct Determination of the Chirality of Organic Molecules by Scanning Tunneling Microscopy
}

\author{
Hongbin Fang, Leanna C. Giancarlo, and George W. Flynn* \\ Department of Chemistry and Columbia Radiation Laboratory, Columbia University, \\ New York, New York 10027
}

Received: April 21, 1998; In Final Form: June 20, 1998

\begin{abstract}
Scanning tunneling microscopy (STM) has been used to study the chiral molecules $(R) /(S)$-2-bromohexadecanoic acid at the liquid/solid interface. When adsorbed onto the basal plane of graphite, these molecules segregate on the surface into domains of pure $R$ or $S$ enantiomers. The atomic resolution obtained in the STM images of these species allows a direct assignment of the chirality of individual molecules.
\end{abstract}

\section{Introduction}

Chirality is a very important phenomenon in nature. Ever since Pasteur's separation of the enantiomers of sodium tartrate using optical microscopy, many physical and chemical methods (polarimetry, ${ }^{1} \mathrm{X}$-ray crystallography, ${ }^{2}$ circular dichroic spectroscopy, ${ }^{3} \mathrm{NMR},{ }^{4}$ etc.) have been used to determine the configuration of chiral molecules. Most of these methods, however, rely on a comparison to a standard sample or make use of compounds with known chirality to react with the unknown sample in order to elucidate chirality. Here, we present an example in which scanning tunneling microscopy (STM) has been utilized to determine the absolute configuration of organic molecules with a single chiral carbon atom, $(R) /(S)$ 2-bromohexadecanoic acid $\left(\mathrm{CH}_{3}\left(\mathrm{CH}_{2}\right)_{13} \mathrm{CHBrCOOH}\right)$, directly.

$\mathrm{STM}^{5}$ along with the whole family of scanning probe microscopies (SPM's), has been widely used to study bare surfaces and various atoms and molecules adsorbed on surfaces. In some cases, ${ }^{6,7}$ atomic resolution has been achieved such that the relative position of individual atoms in a molecule can be clearly observed. Given this sensitivity, SPM seems to be a promising method for determining the absolute configuration of chiral molecules. Indeed some progress has been made in this direction. By using atomic force microscopy (AFM), a few chiral symmetry breaking phenomena in Langmuir-Blodgett films have been observed. ${ }^{8,9} R$ and $S$ enantiomers of chiral biphenylbenzoates have been found by STM to give different 2-D chiral images; however, the molecular resolution obtained in these studies did not allow the direct assignment of the absolute configuration of the individual enantiomers. ${ }^{10,11} \mathrm{Re}-$ cently, there has been a beautiful demonstration of the principles of chiral HPLC using chemical force microscopy. ${ }^{12}$ By attaching molecules with known chirality to the probe tips, two enantiomers of mandelic acid have been distinguished through differences in both adhesion forces and frictional forces. In the study presented here, atomically resolved STM images of 2-bromohexadecanoic acid adsorbed on a graphite surface provide a direct assignment of single molecules as having $R$ or $S$ chiral centers directly through the use of STM "marker" chemical groups and atoms.

* To whom correspondence should be addressed.

\section{Experimental Section}

An enantiomeric mixture of 2-bromohexadecanoic acid $\left(\mathrm{CH}_{3}\left(\mathrm{CH}_{2}\right)_{13} \mathrm{CHBrCOOH}\right)$ was obtained from Aldrich and used without further purification. This sample was dissolved in 1-phenyloctane (Aldrich) to produce a nearly saturated solution. A drop of solution was applied directly to the basal plane of a freshly cleaved piece of highly oriented pyrolytic graphite (HOPG, Advanced Ceramics Corporation).

All STM experiments were performed on a Nanoscope III (Digital Instruments) with mechanically cut Pt/Rh (87/13) wire tips (Omega) immersed in solution. The images were collected under ambient conditions at room temperature. Typical tunneling parameters used for imaging these molecules are $0.8-$ $1.4 \mathrm{~V}$ (sample negative) and 240-400 pA. Different tips and samples were used to check for reproducibility and to ensure there are no image artifacts caused by the tip or sample. All of the images have been acquired at constant current and display the relative topographic height as the tip is raster-scanned across the sample. Here, "bright" areas correspond to topographically higher regions, while "dark" areas reflect lower ones.

Molecular models were created with Biosym (InsightII, Molecular Simulations, Inc.) software running on a Silicon Graphics workstation.

\section{Results and Discussion}

The molecular structures of the molecules $(R) /(S)$-2-bromohexadecanoic acid $\left(\mathrm{CH}_{3}\left(\mathrm{CH}_{2}\right)_{13} \mathrm{CHBrCOOH}\right)$ are schematically shown in Figure 1a,b, where an all-trans conformation, typical of species adsorbed at the liquid-graphite interface, has been assumed. The single chiral carbon on the molecule is indicated by an asterisk.

Upon application of the mixed enantiomer solution to the graphite surface, different domains are formed ranging in width from $30 \mathrm{~nm}$ to more than $100 \mathrm{~nm}$ as measured from STM topographs. These domains appear to be composed of single enantiomers. Although an extensive study of the domain boundaries between the different enantiomers was not made, those that were observed had mirror image structures on either side of the boundary. Parts a and c of Figure 2 represent typical STM images collected from two different domains. These are the only two structures that can be identified on the surface. Despite the difference in resolution between these two images, 
(a)

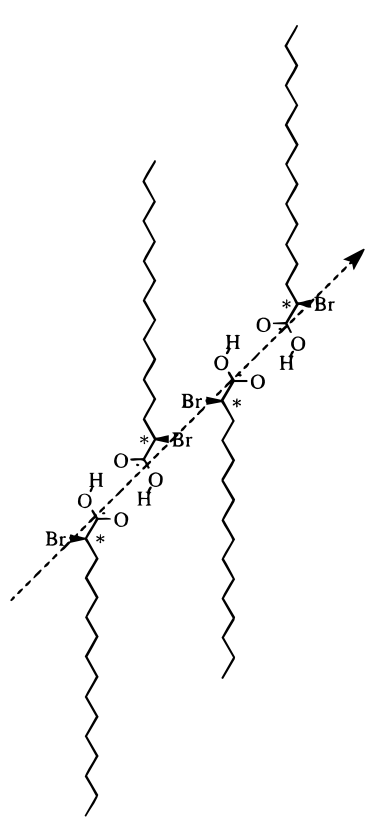

(b)

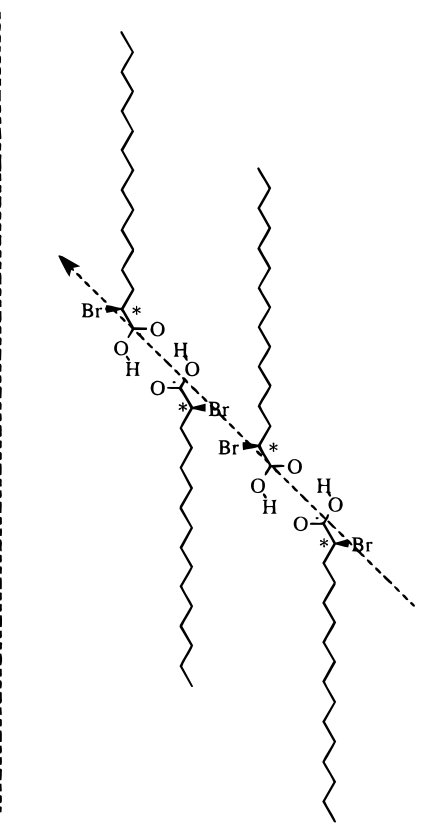

Figure 1. Schematic of the molecular structures of $(R)$-2-bromohexadecanoic acid (a) and ( $S$ )-2-bromohexadecanoic acid (b) physisorbed on a graphite surface with an all-trans conformation. The single chiral carbon atom on the molecules is indicated by an asterisk. Each molecule is hydrogen-bonded to the next one through the carboxyl groups. The dashed lines with arrows are just a guide to show the orientation of the lamella. The dashed line between (a) and (b) represents a "mirror", since the structures in (a) and (b) are mirror images of each other.

it is apparent that these topographs reflect mirror images of each other. This fact is even more evident upon inspection of the angle from the long molecular axis to the direction of the lamellar rows. This angle is about $45^{\circ}$ clockwise for molecules in Figure $2 \mathrm{a}$, while it is $45^{\circ}$ counterclockwise for molecules in Figure 2c.

Figure $2 \mathrm{a}$ is a $12 \times 12 \mathrm{~nm}^{2}$ STM image taken at the interface of the phenyloctane solution and graphite. A computergenerated molecular model deduced from this image is shown in Figure 2b. There are two dominant features in this image: lines (marked as $\mathrm{L}$ in the figure) with alternating bright and dark segments and strips (marked as S) of small spots between these lines. On the basis of the molecular structure of 2-bromohexadecanoic acid shown in Figure 1 and previous STM studies, ${ }^{6,7,13}$ each black bar in the image is attributed to one molecule lying flat on the surface adopting an all-trans conformation. The length of the molecule measured in the STM image is $2.38 \pm 0.06 \mathrm{~nm}$, which matches the length of the molecule with an all-trans conformation $(2.24 \mathrm{~nm})$. Each molecular image is composed of three features: a large bright spot, a dark part adjacent to the bright spot, and a chain of a few small protrusions attached to the bright and dark features. This picture is much clearer in Figure 3, which is an enlargement of the area bounded by the rectangular box in Figure 2a. Previous studies on long chain- $n$-carboxylic acids ${ }^{6,7}$ have shown that these molecules physisorb on graphite with the carboxyl end groups oriented toward each other in order to facilitate hydrogen bonding. These studies also reveal that the carboxyl end group always appears with "darker" contrast compared to the rest of hydrocarbon chain. The dark part in the image can, thus, be ascribed unambiguously to the carboxyl groups, marked as $-\mathrm{COOH}$ in Figure 3. We attribute the large "bright" spot (marked as $\mathrm{Br}$ in the figure) to the location of the bromine atom on the basis of a comparison to earlier work in this laboratory. ${ }^{13}$

Given the relative position of the bromine atom and the carboxyl group, the orientation of the rest of the alkyl chain is determined, since the bromine atom is between the carboxyl group and the rest of the alkyl chain (as can be seen in the molecular structures depicted in Figure 1). The long alkyl tail of the molecule must lie down on the opposite side of the bromine atom from the carboxyl group. Thus, the alkyl chain associated with the lower of the two labeled bromine atoms (marked as $\mathrm{Br}$ in the figure) is represented by the lower set of numbers 1-14 in Figure 3. Atomic resolution has been achieved in this image. There are a few small protrusions visible on most molecules. Experimental and theoretical studies ${ }^{14}$ of $n$-alkanes have assigned the small spots in such STM images to the positions of those hydrogen atoms of the adsorbed hydrocarbon molecules protruding farthest out of the molecular plane. In a similar manner we attribute the small spots (numbered 1-14 in Figure 3) to the hydrogen atoms on the methylene carbons of 2-bromohexadecanoic acid. The spacings between these small protrusions match very well with those of hydrogen atoms on the methylene carbons of 2-bromohexadecanoic acid when it assumes an alltrans conformation (the spacings between the two closest protrusions along each row are $0.26 \pm 0.01 \mathrm{~nm}$ and those between the protrusions in adjacent rows are $0.26 \pm 0.02 \mathrm{~nm}$ as measured from the STM images; the spacings obtained from crystallographic data are $0.25 \mathrm{~nm}$ for both). There are a few small protrusions missing on many molecules. This presumably results from a Moiré pattern present in the image, which will be discussed later.

On the basis of the above assignment with bright bromine atoms sticking up, ${ }^{13,15}$ the molecule can be easily identified as the $R$ conformer since the relative positions of three of the four groups attached to the chiral carbon have been directly determined and the fourth (hydrogen atom) is right beneath the bromine atom, as shown in Figure 1a. The atomic resolution obtained in this STM image has enabled us to determine the absolute configuration of a single organic molecule directly, i.e., without the help of any compound of known chirality. Each molecule in Figure 2a can be examined individually, and all are in the same configuration, $(R)$-2-bromohexadecanoic acid.

In the direction parallel to the long molecular axis, there is no contrast change from lamella to lamella, which suggests that the molecules are arranged in a head-to-tail configuration in this direction. The molecules are oriented in a way such that the head (carboxyl group) of one molecule is next to the tail (methyl group) of the next molecule lying along the molecular axis. This can be clearly seen both in the image in Figure $2 \mathrm{a}$ and in the molecular model in Figure 2b. In the direction perpendicular to the long molecular axis, molecules change orientation every other row. The adjacent molecules in this direction lie down side-by-side with their carboxyl group next to a methyl group on the graphite surface. In this way, a molecule in one column can hydrogen bond to a molecule in the next column and form a dimer (marked as two black bars in the image). This dimer is also a chiral structure with a mirror image that is different from itself. This chiral dimer acts as a building block for ordering in the two-dimensional thin film and forms the overall chiral structure observed by STM. Another interesting feature is that the bromine atom, the large bright spot in the STM image, is always next to another bromine atom forming a twin structure. Recent studies ${ }^{13}$ on related bromine-substituted long-chain hydrocarbon molecules have described a similar phenomenon apparently arising from strong $\mathrm{Br} / \mathrm{Br}$ van der Waals interactions.

There is also a Moiré pattern present in Figure 2a, which 
(a)

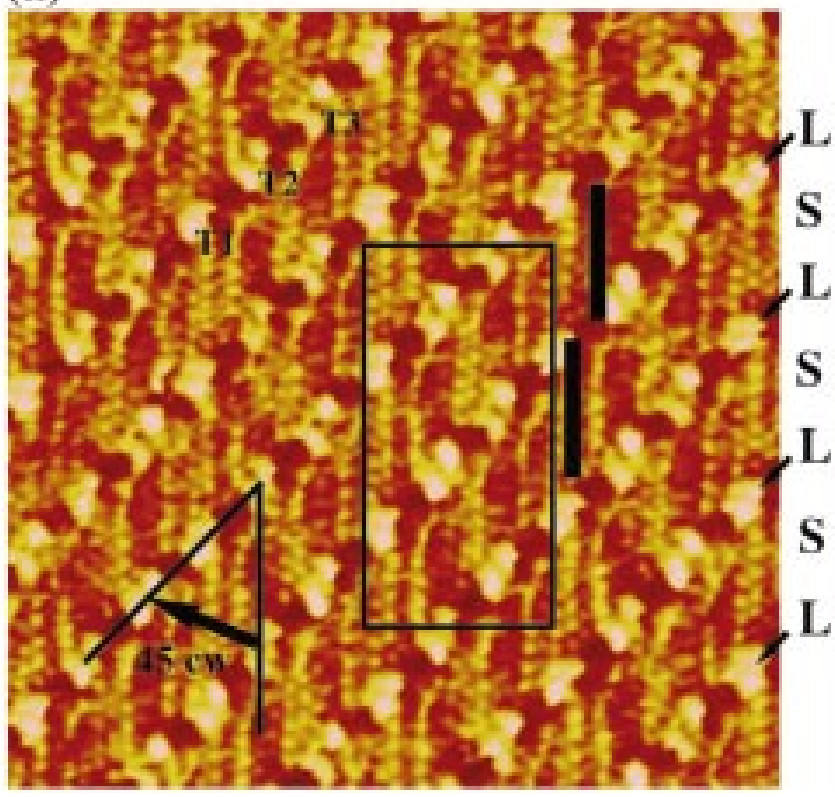

(c)

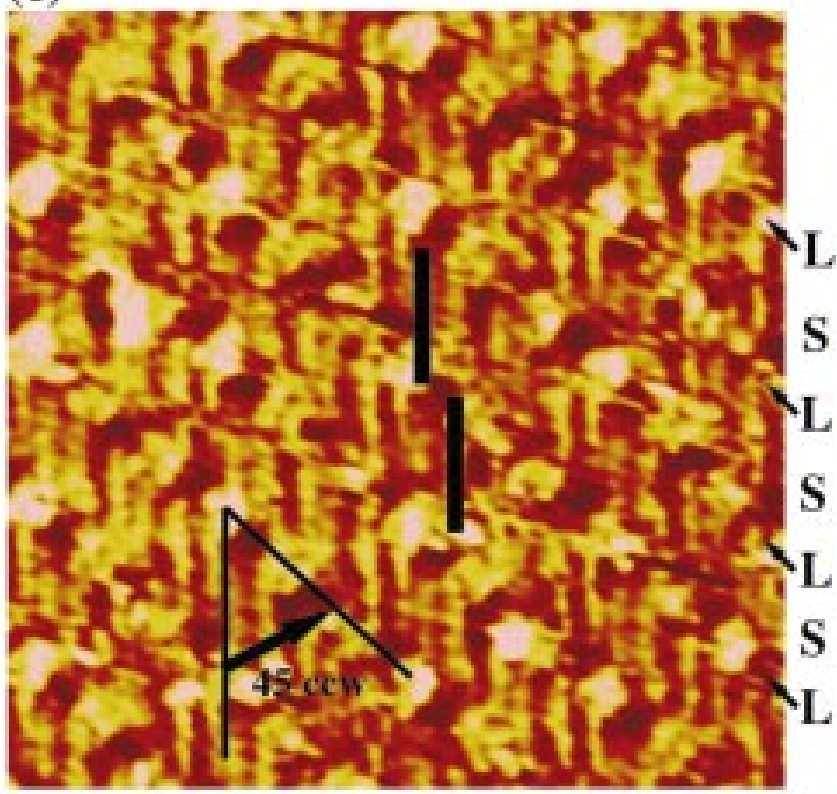

(b)

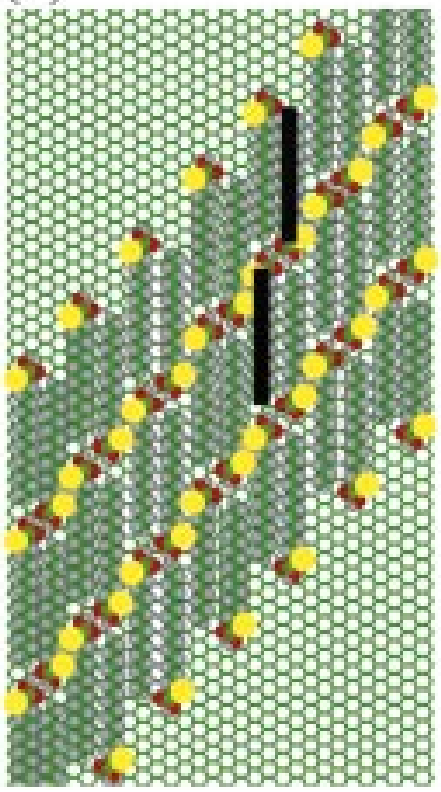

(d)

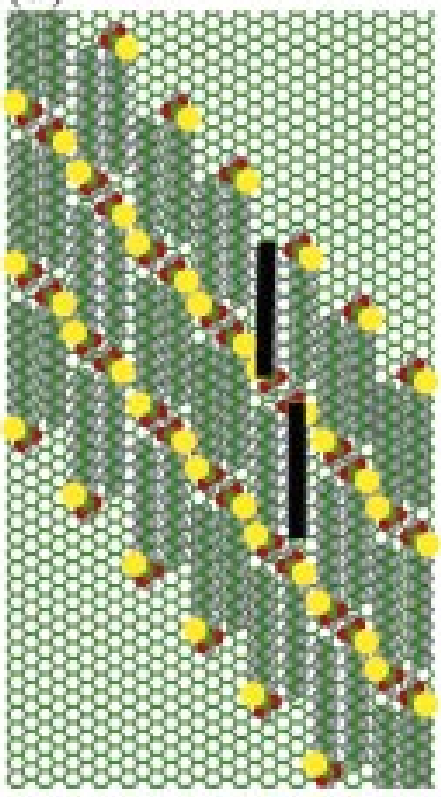

Figure 2. (a) $12 \times 12 \mathrm{~nm}^{2}$ STM constant-current image of $(R)$-2-bromohexadecanoic acid at the interface of a phenyloctane solution and the graphite basal plane. One molecular length is indicated by a thick black bar. Two thick black bars correspond to the chiral dimer formed by two molecules hydrogen-bonded through carboxyl groups (see Figure 1a). The $45^{\circ}$ angle "clockwise" is from the direction of the long axis of the molecule to the direction of the lamellar orientation. Capital letter L points to lines with alternating bright and dark segments, and S refers to the strips of small spots between the lines. Capital letters T1, T2, and T3 mark the positions of three consecutive twin structures. The rectangular box surrounds an area blown up to show detailed structures in Figure 3. The imaging parameters are $1.4 \mathrm{~V}$ (sample negative) and $300 \mathrm{pA}$. (b) Top view of a model of $(R)$-2-bromohexadecanoic acid molecules on a graphite surface. Yellow represents bromine atoms, green represents carbon atoms, gray represents hydrogen atoms, and red represents oxygen atoms. Two black bars mark the chiral dimer formed by two molecules hydrogenbonded through the carboxyl groups. (c) $12 \times 12 \mathrm{~nm}^{2}$ STM image of (S)-2-bromohexadecanoic acid at the interface of a phenyloctane solution and the graphite basal plane. One molecular length is indicated by a thick black bar. Two black bars correspond to the chiral dimer formed by two molecules hydrogen-bonded through carboxyl groups. The $45^{\circ}$ angle "counterclockwise" is from the direction of the long axis of the molecule to the direction of the lamellar orientation. Capital letter L points to lines with alternate bright and dark segments, and S refers to the strips of small spots between the lines. The imaging parameters are $1.4 \mathrm{~V}$ (sample negative) and $300 \mathrm{pA}$. (d) Top view of a model of $(S)$-2-bromohexadecanoic acid molecules on a graphite surface. Two black bars mark the chiral dimer formed by two molecules hydrogen-bonded through carboxyl groups. gives contrast variation across the image. The period of this pattern repeats every six molecules in the direction perpendicular to the long molecular axis. Previous studies $6,7,16$ have reported a similar Moiré pattern for $n$-carboxylic acids adsorbed on graphite. In the direction perpendicular to their long molecular axes, arachidic acid $\left(\mathrm{C}_{19} \mathrm{H}_{39} \mathrm{COOH}\right)$ and stearic acid $\left(\mathrm{C}_{17} \mathrm{H}_{35^{-}}\right.$ $\mathrm{COOH})$ molecules form superstructures with periods of four and five molecules, respectively. ${ }^{6,7}$ This results partially from the bulkiness of the carboxyl end group. In order for adjacent carboxyl groups to lie down flat and hydrogen bond to each other, the neighboring molecules are pushed away slightly and no longer fit into the graphite lattice. After a few molecular rows, the carboxylic acid molecules come back into registry with the lattice again. In the present case, in order to hydrogen 


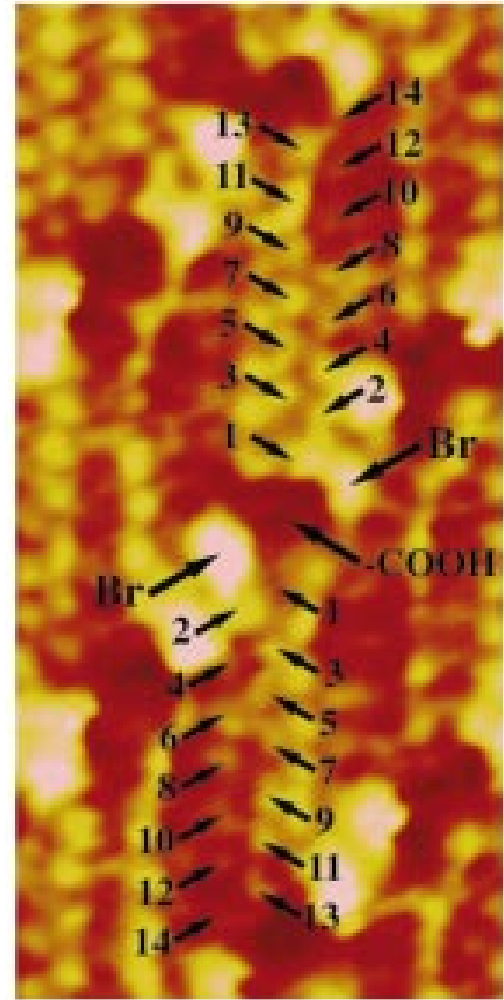

Figure 3. Enlarged image of the area bounded by the rectangular box in Figure 2a. The detailed assignment of individual atoms on a chiral pair of molecules indicated by two black bars in Figure 2a is displayed here. The two $\mathrm{Br}$ designations mark the positions of the bromine atoms, and $-\mathrm{COOH}$ points to the position of the carboxyl groups. Two sets of numbers 1-14 point to the positions of 14 hydrogen atoms on the 14 methylene carbons of the two molecule extending away from the $-\mathrm{COOH}$ groups.

bond with the next molecule and bring two bromine groups together, molecules not only form a Moiré pattern on the graphite surface but also shift in the direction along their long molecular axes to achieve a stronger interaction and form a more stable interfacial film. Thus, as a result of the interplay between the competing adsorbate-adsorbate and adsorbate-substrate interactions, 2-bromohexadecanoic acid forms a structure similar, but not identical, to the corresponding unsubstituted carboxylic acid, $n$-hexadecanoic acid. ${ }^{6,7}$

The atomic resolution obtained in Figure $2 \mathrm{a}$ also allows some subtle variations in the image to be detected. The relative positions of the bright spots in the $\mathrm{Br} / \mathrm{Br}$ twin structures change every three twins (six molecules) which coincide with the Moiré pattern period along the lines (L). The two bright spots (marked as T1 in the figure; the measured distance between them is 0.42 $\pm 0.04 \mathrm{~nm}$ ) in one of the three consecutive twin structures are closer to each other than those in the other two twin structures (marked as T2 and T3 in the figure, with a spacing between them of $0.50 \pm 0.02 \mathrm{~nm}$ ). As 2-bromohexadecanoic acid forms a Moiré pattern having a period of six molecules on the graphite surface, these six molecules take up the width of 13 columns on the graphite lattice ${ }^{6,17}$ (as seen in Figure $2 b$ ), while six unsubstituted alkane molecules only take up 12 columns. ${ }^{16}$ The first two molecules (T1) presumably fit well into the graphite lattice. In order for more molecules to fit comfortably into the graphite lattice, the remaining four molecules are not only pushed away slightly from their lattice positions in the direction perpendicular to their long axes but also shift in a direction parallel to the long molecular axes by a small amount. This separates the bromine groups from each other more in the later four molecules than in the first two. All the above features have been included in the model shown in Figure $2 b$. The essential molecular geometry of this model is shown in Figure 1a. The dashed line with an arrow is just a guide for the eye along the chiral lines (L) mentioned earlier. This model has been constructed with each molecule lying flat on the graphite surface and with the bromine atom pointing up.

Figure $2 \mathrm{c}$ is a $12 \times 12 \mathrm{~nm}^{2} \mathrm{STM}$ image taken from another area of the surface. The resolution of this image is not as good as that attained in Figure 2a, but the important features of the two-dimensional structure can still be readily discerned. Again, there are two parts to the image: lines (marked as $\mathrm{L}$ in the figure) consisting of alternating bright and dark segments and strips (marked as S) of small spots between those lines. Each black bar in the image corresponds to one molecule lying flat on the surface in an all-trans conformation. Each molecular image is composed of three parts: a large bright spot, $\mathrm{Br}$, next to a dark region, the carboxyl group, and a chain of a few small protrusions. Each molecule hydrogen bonds to another one in the next column and forms a dimer (marked as two black bars in the image), which is a chiral structure as well (see Figure 1b). There is also a Moiré pattern with a period of six molecules formed in this image. Despite the fact that this image looks very much like the one in Figure 2a, the topographs displayed in parts a and c of Figure 2 are mirror images of each other. The dimers formed in Figure $2 \mathrm{c}$ are mirror images of those formed in Figure 2a. Following the assignment of the locations of the $\mathrm{Br},-\mathrm{COOH}$, and alkyl chain in the same manner as outlined above, all the molecules in the image can be identified as (S)-2-bromohexadecanoic acid. A molecular model of this film is shown in Figure $2 \mathrm{~d}$ for comparison with the images formed by the $\mathrm{R}$ enantiomers.

$R$ and $S$ enantiomers of 2-bromohexadecanoic acid clearly form chiral structures when adsorbed on the graphite basal plane. These chiral structures are mirror images of each other. The atomically resolved STM images have enabled us to "see" the absolute configuration of these molecules on the graphite surface directly, by taking advantage of the bright $\mathrm{Br}$ and dark $-\mathrm{COOH}$ STM "marker" groups. Moreover, the graphite lattice puts additional restrictions on the formation of a stable self-assembled layer structure. When the enantiomers of 2-bromohexadecanoic acid adsorb onto the graphite surface, an $S$ enantiomer only hydrogen bonds to another $S$ enantiomer to form a dimer, and an $R$ enantiomer only hydrogen bonds to another $R$ enantiomer and forms another dimer, which is the mirror image of the one generated by the $S$ enantiomers. This phenomenon observed in the STM images indicates that the interactions between molecules possessing the same chirality are stronger than interactions between molecules having different chirality. If an $R$ enantiomer hydrogen bonds to an $S$ enantiomer, the bromine group on one of the stereoisomers must point down into the graphite surface. When this occurs, the bulky bromine group (both the van der Waals radius of $\mathrm{Br}(1.95 \AA)$ and the $\mathrm{C}-\mathrm{Br}$ bond length $(1.937 \AA$ ) are much larger than the $1.2 \AA \mathrm{H}$ atom van der Waals radius and $1.101 \AA \mathrm{C}-\mathrm{H}$ bond length) lifts the molecule from the graphite surface, thereby decreasing the interactions of the remaining atoms in the molecular chain with the surface. While this structure may occur occasionally, it will eventually be replaced by the more stable dimers in which $R$ enantiomers bond to $R$ enantiomers and $S$ enantiomers bond to $S$ enantiomers. The dimers formed by $S$ enantiomers and those formed by $R$ enantiomers segregate on the graphite surface and form different domains in which mirror image patterns can be easily detected by STM. By examining larger scale STM 
images, e.g. $20 \times 20 \mathrm{~nm}^{2}$, the chirality of the molecules can be simply determined from the overall large-scale packing arrangement of the molecules.

\section{Conclusion}

In summary, it has been clearly demonstrated that STM can be used to determine the chirality of organic molecules directly. The chiral molecules, $(R) /(S)$-2-bromohexadecanoic acid, adsorbed on the graphite surface have been imaged by STM with atomic resolution. These two enantiomers segregate on the graphite surface and form different domains. The two enantiomers give mirror image packing patterns on the graphite surface. The absolute configuration of these molecules can be directly observed in the STM images. The technique described here can be applied to any chiral molecule that self-assembles and forms stable structures on a substrate. In addition three of the four groups and their orientation at the chiral center must be distinguishable in the STM images, as was the case here with bright bromine groups, dark carboxyl groups, and alkyl tails.

Acknowledgment. We thank Professor W. C. Still, Professor R. Breslow, X. Huang, and K. Ryan for several stimulating discussions. Work described here was supported by the National Science Foundation (DMR-94-24296) and by the Joint Services Electronics Program (U.S. Army, Navy, and Air Force; DAAG5597-1-0166).

\section{References and Notes}

(1) Lyle, G. G.; Lyle, R. E. In Asymmetric Synthesis; Morrison, J. D., Ed.; Academic Press: New York, 1983; Vol. 1, p 13.

(2) Lipscomb, W. N.; Jacobon, R. A. In Physical Methods of Chemistry; Rossiter, B. W., Hamilton, J. F., Eds.; Wiley: New York, 1986; Vol. V, p 1 .

(3) Harada, N.; Nakanishi, K. Circular Dichroic Spectroscopy-Exciton Coupling in Organic Stereochemistry; University Science Books: Mill Valley, CA, 1983.

(4) Dale, J. A.; Mosher, H. S. J. Am. Chem. Soc. 1973, 95, 512

(5) Binnig, G.; Rohrer, H.; Gerber, C.; Weibel, E. Phys. Rev. Lett. $1982,49,57$.

(6) Hibino, M.; Sumi, A.; Hatta, I. Jpn. J. Appl. Phys. 1995, 34, 610.

(7) Hibino, M.; Sumi, A.; Hatta, I. Jpn. J. Appl. Phys. 1995, 34, 3354.

(8) Viswanathan, R.; Zasadzinski, J. A.; Schwartz, D. K. Nature 1994, 368,440 .

(9) Eckhardt, C. J.; Peachey, N. M.; Swanson, D. R.; Takacs, J. M.; Khan, M. A.; Gong, X.; Kim, J.-H.; Wang, J.; Uphaus, R. A. Nature 1993, $362,614$.

(10) Stevens, F.; Dyer, D. J.; Walba, D. M. Angew. Chem., Int. Ed. Engl. 1996, 35, 900.

(11) Walba, D. M.; Stevens, F.; Clark, N. A.; Parks, D. C. Acc. Chem. Res. 1996, 29, 591.

(12) McKendry, R.; Theoclitou, M.-E.; Rayment, T.; Abell, C. Nature 1998, 391, 566.

(13) Cyr, D. M.; Venkataraman, B.; Flynn, G. W.; Black, A.; Whitesides, G. M. J. Phys. Chem. 1996, 100, 13747.

(14) Liang, W.; Whangbo, M.-H.; Wawkuschewski, A.; Cantow, H. J.; Magonov, S. N. Adv. Mater. 1993, 5, 817.

(15) Faglioni, F.; Claypool, C. L.; Lewis, N. S.; Goddard, W. A. J. Phys. Chem. B. 1997, 101, 5996.

(16) Rabe, J. P.; Buchholz, S. Science 1991, 253, 424

(17) Hatta, I.; Nishino, J.; Sumi, A.; Hibino, M. Jpn. J. Appl. Phys. 1995, 34, 3930 . 